\title{
Evidence for low-temperature antiferromagnetic phase transition in Ising singlet magnet $\mathrm{KTb}\left(\mathrm{WO}_{4}\right)_{2}$
}

\author{
E. Khatsko ${ }^{1}$, C. Paulsen ${ }^{2}$, and A. Rykova ${ }^{1}$ \\ ${ }^{1}$ Institute for Low Temperature Physics and Engineering of the National Academy of Sciences of Ukraine \\ 47 Lenin Ave., Kharkov 61103, Ukraine \\ E-mail:khatsko@ilt.kharkov.ua \\ ${ }^{2}$ Institut Neel, CNRS, BP 166, F-38042 Grenoble Cedex 9, France
}

Received April 27, 2011

\begin{abstract}
The magnetic susceptibility and magnetization measured along the $b$ axis of a $\mathrm{KTb}\left(\mathrm{WO}_{4}\right)_{2}$ single crystal was investigated experimentally in the temperature range $70 \mathrm{mK}-6 \mathrm{~K}$ and in magnetic fields up to $8 \mathrm{~T}$. The results allow us to make conclusion that singlet magnet $\mathrm{KTb}\left(\mathrm{WO}_{4}\right)_{2}$ undergoes an antiferromagnetic phase transition $T_{c}=0.65 \mathrm{~K}$.

PACS: 75.50.Ee Antiferromagnetics;

75.30.Cr Saturation moments and magnetic susceptibilities;

75.40.Cx Static properties (order parameter, static susceptibility, heat capacities, critical exponents, etc.);

75.25.-j Spin arrangements in magnetically ordered materials (including neutron and spin-polarized electron studies, synchrotron-source x-ray scattering, etc.).
\end{abstract}

Keywords: magnetic susceptibility, magnetization, singlet magnet, phase transition.

Alkali-rare-earth double molybdates and tungstates have been actively studied for a long time. Many compounds of this class are characterized by a strong magnetic anisotropy of the rare-earth ions, a low local symmetry, and a pronounced chain structure. The electronic spectrum of rare-earth ions in the crystalline field usually has lowlying excited levels, and that leads to the possibility of reorganization of the corresponding electronic states both by an external magnetic field and by displacements of the ions. This is responsible, in particular, for the magneticfield-induced structural phase transitions observed in various compounds of this class [1-5]. In the case of nonKramers rare-earth ions the lowest electronic state often form a quasi-doublet with a gap $\Delta$ of the order of few $\mathrm{K}$, well separated from the rest of the spectrum. The magnetic dipole and exchange interactions in these compounds are of the same order of magnitude. Therefore, singlet and excitonic types of magnets can be realized in these compounds (in the second case the interactions are insufficient to induce magnetic order suppressed by the gap $\Delta[6,7]$. In the non-Kramers doublet case the contribution to the magnetic properties of the crystal from the higher-lying excitations of the rare-earth ions are also unusual [6]. All of these circumstances cause great interest to the study of such systems. Here it is possible to study a number of topical questions in solid state physics in a comparatively simple situation. Among such topics are the interaction of electronic excitations with lattice vibrations (the JahnTeller effect, polaron effects, etc.), structural phase transitions taking place by unusual scenarios (incommensurability, strong fluctuations), and nonlinear regimes of microwave energy absorption, which are comparatively easy to achieve here because of the long relaxation times of the elementary excitations $[8,9]$.

One member of this family of compounds that has practically escaped study is $\mathrm{KTb}\left(\mathrm{WO}_{4}\right)_{2}$, which contains the rare-earth ion $\mathrm{Tb}^{3+}\left({ }^{\top} \mathrm{F}_{6}\right)$ with an odd number of electrons.

$\mathrm{X}$-ray studies of the magnet $\mathrm{KTb}\left(\mathrm{WO}_{4}\right)_{2}$ have shown that this compound belongs to the monoclinic class $C_{2} / \mathrm{c}$ with a chain structure [10]. There are 4 formulae units per elementary cell with cell parameters: $a=10.653 \AA, b=$ $=10.402 \AA, c=7.573 \AA, \beta=130.76^{\circ}$. Crystal structure is represented in Fig. 1. The Tb ions form chains along [101] direction, distance between nearest $\mathrm{Tb}^{3+}$ is $4.071 \AA$.

The angular dependence of the magnetic susceptibility [10] shows that at low temperatures a purely Ising aniso- 


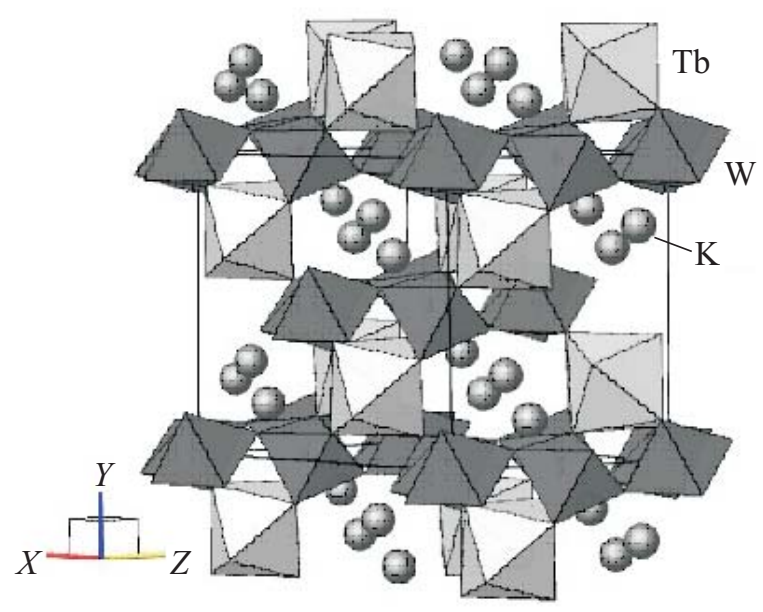

Fig. 1. View of the $\mathrm{KTb}\left(\mathrm{WO}_{4}\right)_{2}$ structure along the [101] direction: the $\mathrm{W}$ polyhedra are dark, the Tb polyhedra are light, and instead of the $\mathrm{K}$ polyhedra only the position of the central atoms are shown. The $X, Y$, and $Z$ axes correspond to the crystallographic axes $a, b$, and $c$ (taken from [10]).

tropy with only one component of the moment along the $b$ axis is realized in this compound. The temperature dependence of the susceptibility has a maximum at $T=1.2 \mathrm{~K}$, but the experimental temperatures were not low enough to make sure that the phase transition exist.

According to resonant studies in $\mathrm{KTb}\left(\mathrm{WO}_{4}\right)_{2}$ [11] the non-Kramers magnetic ion $\mathrm{Tb}^{3+}$ has a quasi-doublet ground state separated by an energy gap $\delta \approx 1 \mathrm{~K}$ which is well separated from the rest of the spectrum. The magnetic dipole and exchange interactions are of the same order.

The rare-earth compounds with non-Kramers magnetic ions often belong to the class of so-called singlet magnets, which can exhibit unusual magnetic properties because of the presence of a rather large energy gap between the states of the quasi-doublet. As the relationship between the values of the gap $\delta$ and the magnetic interactions $I$ varies, the magnetic properties of these compounds undergo radical changes. In particular, there exists a critical value of the ratio $\delta / I$ above which spontaneous magnetism becomes impossible even at zero temperature. However, this critical value depends substantially on the properties of the interactions and foremost, on the character of their spatial anisotropy.

The goal of the present study was to look for the existence of a magnetic phase transition and, if it exists, investigate its features.

The measurements were carried out with a SQUID magnetometer developed at the Institute Néel equipped with a miniature ${ }^{3} \mathrm{He}-{ }^{4} \mathrm{He}$ dilution refrigerator allowing measurements down to $70 \mathrm{mK}$.

As the nonzero magnetic moment exist only along one axis $b$ [10], we investigate magnetic properties along this axis. The magnetic susceptibility along the $b$ axis of $\mathrm{KTb}\left(\mathrm{WO}_{4}\right)_{2}$ single crystal was investigated experimentally

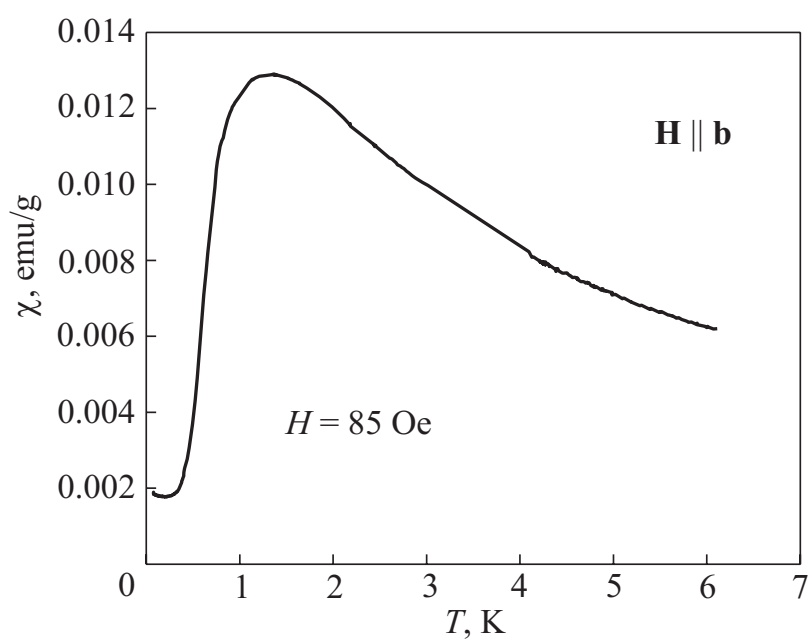

Fig. 2. Temperature dependence of the magnetic susceptibility of the $\mathrm{KTb}\left(\mathrm{WO}_{4}\right)_{2}$ single crystal along the $b$ axis.

in the temperature range $70 \mathrm{mK}-6 \mathrm{~K}$ in small magnetic field of 85 Oe. The results are presented in Fig. 2. The temperature dependence of the susceptibility has a maximum at $T=1.2 \mathrm{~K}$. Above this temperature $\chi(T)$ is described by Curie-Weiss law with negative (antiferromagnetic) Curie temperature $\Theta=-2 \mathrm{~K}$. Below $1.2 \mathrm{~K}$ the susceptibility drops sharply and below $0.4 \mathrm{~K}$ become practically independent on temperature. Such behavior is typical for a magnetic phase transition in the antiferromagnetic state and is described by mean field theory for singlet magnet [10]. The transition temperature was determined from the maximum of derivate $d \chi / d T$ is $T_{c}=0.65 \mathrm{~K}$.

The magnetization curves $M(H)$ along the $b$ axis were studied in magnetic field up to $8 \mathrm{~T}$ in temperature range $80 \mathrm{mK}-4.2 \mathrm{~K}$. The results are shown in Fig. 3. The saturation magnetic moment is $8.9 \mu_{B}$, which is somewhat smaller than the calculated values for $\mathrm{Tb}^{3+}$ ion $9.7 \mu_{B}$.

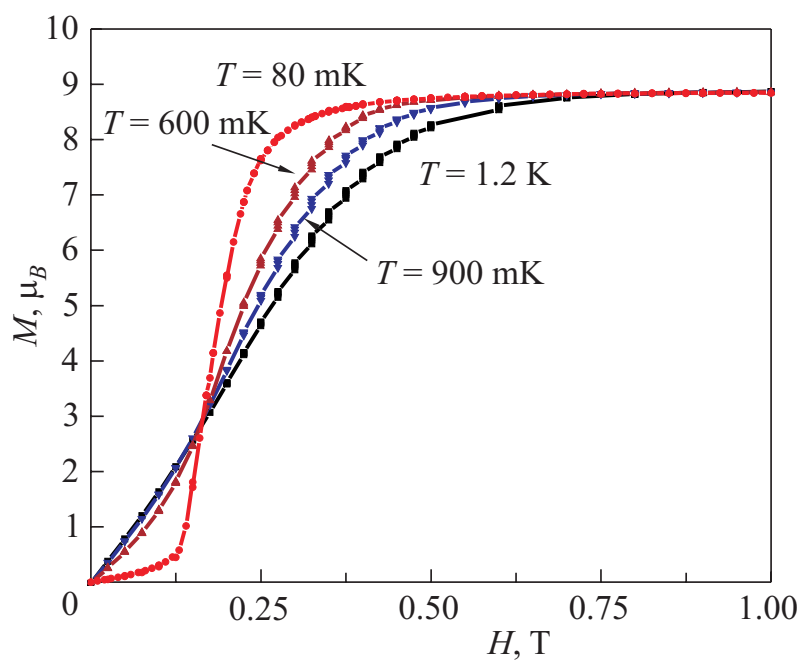

Fig. 3. Field dependence of magnetization of the $\mathrm{KTb}\left(\mathrm{WO}_{4}\right)_{2}$ single crystal along the $b$ axis. 
At low temperatures a nonlinear magnetic behavior was found, which we explain as the metha-magnetic phase transition in magnetic field near $0.2 \mathrm{~T}$. Such a transition can take place when a crystallographic anisotropy is much larger than the exchange. With increasing temperature the nonlinearity of magnetization decreases and disappears above $600 \mathrm{mK}$. This is consistent with the antiferromagnetic transition temperature determined from the susceptibility data.

All obtained results allow us to make conclusion that the singlet magnet $\mathrm{KTb}\left(\mathrm{WO}_{4}\right)_{2}$ undergoes antiferromagnetic phase transition with collinear magnetic structure at $T_{c}=0.65 \mathrm{~K}$.

In addition we observe the field induced methamagnetic phase transition in small magnetic field $0.2 \mathrm{~T}$.

1. M.J.M. Leask, A.C. Tropper, and M.R. Wells, J. Phys. C: Solid State Phys. 14, 3481 (1981).

2. E.N. Khatsko, Yu.V. Pereverzev, M.I. Kobets, V.A. Pashchenko, and V.I. Kut'ko, Fiz. Nizk. Temp. 21, 1061 (1995) [Low Temp. Phys. 21, 816 (1995)].
3. E.N. Khatsko, M.I. Kobets, V.I. Kutko, and V.A. Pashchenko, Ferroelectrics 175, 73 (1996).

4. E.N. Khatsko, M.I. Kobets, and Ju.V. Pereverzev, Ferroelectrics 233, 93 (1999).

5. E.N. Khatsko, M.I. Kobets, and Ju.V. Pereverzev, Bull. Magn. Reson. 19, 56 (1999).

6. A.K. Zvezdin, V.M. Matveev, A.A. Mukhin, and A.I. Popov, Rare-Earth Ions in Magnetically Ordered Crystals, Nauka, Moscow (1985) (in Russian).

7. L. Nagaev, Magnets with Composite Exchange Interactions, Nauka, Moscow (1988) (in Russian).

8. V.I. Kut'ko and M.I. Kobets, Fiz. Nizk. Temp. 22, 1447 (1996) [Low Temp. Phys. 22, 1099 (1996)].

9. M.I. Kobets, Fiz. Nizk. Temp. 26, 96 (2000) [Low Temp. Phys. 26, 72 (2000)].

10. A.A. Loginov, E.N. Khatsko, A.S. Cherny, V.N. Baumer, A.I. Rykova, P.S. Kalinin, and A. Sulpis, Fiz. Nizk. Temp. 32, 91 (2006) [Low Temp. Phys. 32, 68 (2006)].

11. K.G. Dergachev, M.I. Kobets, A.A. Loginov, and E.N. Khatsko, Fiz. Nizk. Temp. 31, 1130 (2005) [Low Temp. Phys. 31, 862 (2005)]. 